\title{
Editorial: The Evolution of Interdisciplinary Research on Human Behavior, Brain, and Body
}

\author{
Dario Maestripieri
}

Published online: 27 March 2014

(C) Springer International Publishing 2014

The scientific study of human behavior has traditionally been the subject of many different disciplines such as anthropology, biology, economics, psychiatry, psychology, and sociology. For a long time, researchers within each of these disciplines worked in isolation from others, such that each discipline had its own set of theories, methods, results, and explanations. Although conceptual and methodological boundaries between disciplines still exist, more behavioral scientists working in the 21 st century are crossing these boundaries than ever before.

Psychologists and biologists have been particularly active in establishing interdisciplinary bridges, but this has occurred only in recent history. In the early decades of the 20th century, there was only one main bridge connecting the study of human behavior in psychology and biology, although this bridge had two different names, depending on the direction in which it was crossed. Psychologists called it biological psychology, or biopsychology, while in biology it was known as psychobiology. But in the last 50 years, biopsychology has evolved and diversified into a number of new neuroscience subdisciplines (behavioral, social, affective, cognitive, and cultural neuroscience, among others), while psychobiology has hybridized with other branches of biology and produced even more subdisciplines such as psychophysiology, psychoneuroendocrinology, psychoimmunology, and behavioral genetics. Among the latest arrivals in this evolutionary process of discipline "speciation" are subdisciplines focusing on the biological substrates of social relationships such as social neuroscience and social neuroendocrinology, reflecting a historical shift in interest in the human behavioral sciences, from the study of learning in the laboratory to that of social processes in the real world.

Although psychology and biology have led the way in the evolution of interdisciplinary studies of human behavior, this process has also involved other behavioral disciplines such as anthropology, economics, and psychiatry giving rise to new subdisciplines such as neuroanthropology, human biology and ecoimmunology, neuroeconomics, and biological psychiatry. As is the case of subdisciplines in psychology and biology, the range of action of these disciplines is not limited to behavior, but

D. Maestripieri $(\bowtie)$

Department of Comparative Human Development and Institute for Mind and Biology, The University of Chicago, Chicago, IL, USA

e-mail: Dario@uchicago.edu 
also includes other biological processes (e.g., growth, reproduction, and health), cognitive and affective processes, and social-cultural factors.

Evolutionary processes in nature are triggered by genetic mutation and recombination in conjunction with changes in the environment. Evolutionary processes in scientific research, instead, are primarily driven by advances in theories and in technology, along with changes in the environment in which researchers operate. It is undeniable that a primary force in the evolution of interdisciplinary studies of human behavior has been the development of new technologies for the assessment of brain function and physiological, molecular, and genetic processes as well as a much better understanding of the reciprocal interactions between brain, body, behavior (the $3 \mathrm{Bs}$ ), genes, and environment. In terms of theoretical advances, a major factor has been the systematic application of Darwinian evolutionary theory to the study of animal and human behavior, which began in the 1960s and gained momentum in the 1970s and 1980s. It may be argued that the famous statement by evolutionary biologist Theodosius Dobzhanski that "nothing in biology makes sense except in the light of evolution" also applies to the 3Bs of behavior, brain, and body. In fact, the last few decades have seen the rise of new evolutionary behavioral disciplines such as sociobiology, human ethology, human behavioral ecology, and evolutionary psychology, whose main goal is to understand the adaptiveness of human behavior, that is the extent to which behavior has evolved by natural selection to maximize survival and reproduction in response to changes in the environment. On the basis of Darwinian evolutionary theory and its "secondary theories" (e.g. life-history theory, parental investment theory, kinship and altruism theory, and sexual selection theory) major conceptual advances have been made in our understanding of fitness-relevant behavioral processes such as aggression and competition for status and resources; affiliation, bonding, and cooperation; sexual attraction, courtship, and mating; parent-child attachment; and development and life-history.

Evolutionary studies addressing the adaptiveness of human behavior can proceed independently from studies addressing the genetic, physiological, and neurobiological regulation of behavior. Modern evolutionary studies of human behavior, however, often include specific hypotheses about the genetic, neural, and neuroendocrine substrates that evolved by natural or sexual selection to support fitness-relevant cognitive, affective, or behavioral outcomes. Therefore, evolutionary theory can guide and inform research on proximate mechanisms. This point has been forcefully made by evolutionary psychologists, who have made a central part of their research agenda the study of the neural architecture of mental and behavioral processes. Integrating the study of adaptive function with that of mechanisms has led to major breakthroughs in our understanding of the relationship between behavior, brain and body, including, just to give a few examples, the neural circuitry underlying financial risk-taking and decisionmaking; the neural and neuroendocrine substrates of empathy and prosocial behavior; the influence of neurotransmitter systems in the regulation of fear, aggression, impulsivity and reward-seeking; the influence of male and female sex steroid hormones on sexual attractiveness, arousal, motivation, and courtship behavior; the role of adrenal and gonadal hormones in the regulation of competition and dominance and submission; the role of neuropeptides in the regulation of social bonds and parental behavior; the interaction between the HPA axis, the HPG axis, and the immune and cardiovascular systems; the role of early experience in calibrating the development of stress reactivity; 
and the interaction between allelic variation, reactivity to the environment, and behavioral and reproductive life-history traits.

Although behavior is ultimately the product of biological processes that reside in the brain, there is no simple or direct association between particular regions of the brain and particular aspects of behavior. Different areas of the brain are structurally and functionally interconnected, and the brain has a two-way communication system with the rest of the body via the autonomic nervous system, the endocrine system, the immune system and the cardiovascular system. Moreover, behavior, brain, and body are all influenced by the same genetic information contained in an individual's DNA. In theory, research on genetic or neural regulation of behavior could proceed independently from research in behavioral neuroendocrinology or psychoimmunology. This independence of approaches is reflected in the existence of journals that specialize in genes and behavior, or brain function and behavior, or endocrine function and behavior, or immune function and behavior. This compartmentalization of research, however, is neither necessary nor productive. Since behavior, brain and body interact in complex ways, research on different aspects of the 3Bs could and should be integrated.

The evolution of interdisciplinary research on human behavior, brain, and body has now reached a stage in which there is greater need for empirical work to be driven and guided by comprehensive theories that have validity across species and levels of analyses, as well as greater need for integration of work conducted in different disciplines and subdisciplines. My hope for Adaptive Human Behavior and Physiology is that this new scientific journal will make a useful contribution to the further evolution of this field of research. The journal's mission is to publish theoretical and empirical studies of any aspects of adaptive human behavior (e.g., cooperation, affiliation, and bonding, competition and aggression, sex and relationships, parenting, decision-making), with an emphasis on studies that also address the biological (e.g. neural, endocrine, immune, cardiovascular, genetic) mechanisms controlling behavior. The journal welcomes research on behavior in relation to cognition, emotion, stress, personality, early development, growth, reproduction, relationships, health, and aging. Physiological mechanisms are broadly conceived to include brain function, neurotransmitters, hormones, immune function, the autonomic nervous system, and the cardiovascular system; genetic control of behavior is also included.

Submissions are encouraged from all scientific disciplines investigating the biological regulation of human behavior including evolutionary biology, behavioral and social ecology, behavioral and social endocrinology, behavioral and social neuroscience, ethology and neuroethology, behavioral genetics, biological and evolutionary anthropology, psychophysiology, psychoneuroendocrinology and psychoneuroimmunology, behavioral economics and neuroeconomics, and developmental, cognitive, social, and evolutionary psychology. Please visit the journal's website (www.springer.com/40750) and consider Adaptive Human Behavior and Physiology as an outlet for your best scientific publications. 